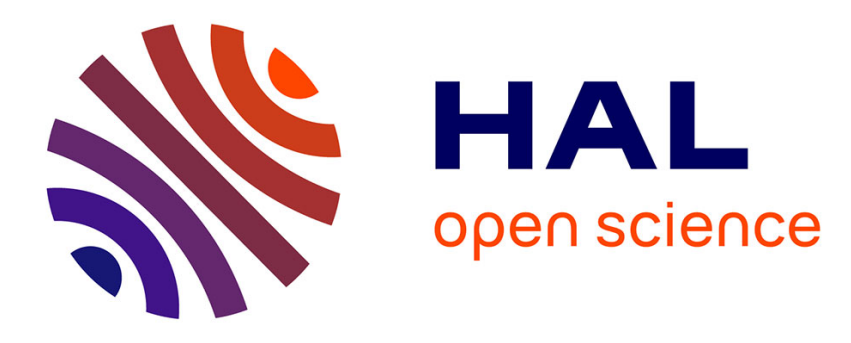

\title{
Boundary Control Design for Traffic with Nonlinear Dynamics
}

Liudmila Tumash, Carlos Canudas de Wit, Maria Laura Delle Monache

\section{To cite this version:}

Liudmila Tumash, Carlos Canudas de Wit, Maria Laura Delle Monache. Boundary Control Design for Traffic with Nonlinear Dynamics. IEEE Transactions on Automatic Control, 2022, 67 (3), pp.13011313. 10.1109/TAC.2021.3069394 . hal-02955853

\section{HAL Id: hal-02955853 https://hal.science/hal-02955853}

Submitted on 2 Oct 2020

HAL is a multi-disciplinary open access archive for the deposit and dissemination of scientific research documents, whether they are published or not. The documents may come from teaching and research institutions in France or abroad, or from public or private research centers.
L'archive ouverte pluridisciplinaire HAL, est destinée au dépôt et à la diffusion de documents scientifiques de niveau recherche, publiés ou non, émanant des établissements d'enseignement et de recherche français ou étrangers, des laboratoires publics ou privés. 


\title{
Boundary Control Design for Traffic with Nonlinear Dynamics
}

\author{
Liudmila Tumash, Carlos Canudas-de-Wit, Fellow, IEEE and Maria Laura Delle Monache, Member, IEEE
}

\begin{abstract}
We address a problem of boundary control for a nonlinear scalar conservation law. Namely, this paper is devoted to the boundary control of an LWR PDE with triangular flux function evolving along a single road. The target state is a time- and space-dependent trajectory. The boundary control law is constructed using the analytical solution of the Hamilton-Jacobi equation, which is an integral form of the LWR PDE. We design a feedback controller and illustrate its performance on a numerical example using the Godunov scheme.
\end{abstract}

Index Terms-boundary control problem, HamiltonJacobi formulation, LWR model, partial differential equations, traffic control.

\section{INTRODUCTION}

\section{A. Motivation and Literature Review}

C ONSERVATION laws are a very important topic to study nowadays, since a significant part of physical systems is based on the principle of the conservation of some quantity, for example, mass or energy. Urban and highway traffic behaviour is modeled via conservation laws with the principle of preserving the number of cars. The first work describing the behaviour of traffic on a macroscopic scale was proposed by Lighthill, Whitham [2], and, independently, Richards [3] in the fifties (LWR model). The LWR model is a first-order model based on the conservation of mass equation. Despite the appearance of more sophisticated first order [14], [16], [17] or even higher order models capable of covering more realistic traffic behaviour [13], [18], the LWR model remains the most used one to study traffic behaviour due to its simplicity. Mathematically, the LWR model is a hyperbolic scalar conservation law with a nonlinear concave flux function, which represents an empirical relation between flow and density, see [25] for a detailed review.

Traffic congestion represents a considerable issue worldwide that negatively impacts traffic mobility, especially in large urban areas, which makes traffic management an important

Manuscript received October 24, 2019; revised October 1, 2020. This work is a part of Scale-FreeBack project that received funding from the European Research Council (ERC) under the European Unions Horizon 2020 research and innovation programme (grant agreement N 694209).

L. Tumash is with Univ. Grenoble Alpes, CNRS, Inria, Grenoble INP, GIPSA-lab, 38000 Grenoble, France (email: liudmila.tumash@gipsalab.fr).

C. Canudas-de-Wit is with Univ. Grenoble Alpes, CNRS, Inria, Grenoble INP, GIPSA-lab, 38000 Grenoble, France (email: carlos.canudas-dewit@gipsa-lab.grenoble-inp.fr).

M. L. Delle Monache is with Univ. Grenoble Alpes, Inria, CNRS, Grenoble INP, GIPSA-lab, 38000 Grenoble, France (email: ml.dellemonache@inria.fr). subject. Some examples of control with application to traffic can be found in [20], [30]. Traffic flow control does not require changes in the topology of urban traffic network and can be applied directly provided that there is some knowledge about current traffic situation. Being modeled by conservation laws, traffic systems are usually controlled by variable speed limit [40], or from the boundary, i.e. either from entry or exit of the road, e.g. ramp metering [37]. Classical techniques widely used for control of conservation laws (without model reduction from PDE to ODE) are backstepping [33], Lyapunov-based [26] and optimal control methods [15], [20], [22] that are used to achieve minimization of total travel time and of fuel consumption or maximization of the vehicle outflow.

It is quite challenging to control hyperbolic conservation laws, since discontinuities (shocks) emerge in finite time even for smooth initial data [12]. The classical control methods mentioned above are not always well suited to handle shocks, since they require the knowledge on the internal shock dynamics. However, shock dynamics inside the domain were tracked, e.g., in [39], where the weak formulation and the Rankine-Hugoniot relation were used to stabilize the solution of the Burgers equation to a constant equilibrium. In a recent work [42] the problem of boundary control of solutions with jump discontinuities has been considered. In both [39], [42], the desired state was stationary and the Lyapunov methods were applied. In [44] the exact controllability of solutions to conservation laws to space- and time-dependent trajectories has been studied. Nevertheless, the problem of stabilizing a state with shocks to space- and time-dependent trajectories that may also contain shocks has never been considered before.

In situations where control design becomes too tedious due to shocks, one can alternatively use the Hamilton-Jacobi formulation that is an integral form of the LWR PDE. The solution of a H-J PDE does not contain any shocks, only discontinuities in its derivatives. The state represents a ситиlated vehicle count, which is a Lipschitz continuous function that can be evaluated directly from the boundary or initial conditions [10]. The existence of such a function guarantees the conservation of the number of vehicles even if shocks arise. The solution of a H-J PDE can be obtained by solving a simple minimization problem, therefore it does not require a model discretization providing exact results. Thus, several computational algorithms based on H-J formulation of LWR model have been developed. A variational formulation of kinematic waves was first proposed in [19], [21], who showed that every well-posed traffic problem with a concave flowdensity relation can be solved as a set of shortest paths. 
The solution of Hamilton-Jacobi PDE can be equivalently obtained using the viability framework [8], [9]. This framework is based on using Lax-Hopf formula that exploits the structure of a dynamic programming problem and the solution is obtained as the minimum of all valid paths [28]. In [29] they develop a computational method of H-J PDE for any piecewise affine initial condition. The Lax-Hopf algorithm for computation of a solution on a single link for any concave fundamental diagram has been addressed in [32]. Its improved version with a lower computational time has been proposed in [41]. Optimal control methods for a traffic network based on viability framework are proposed in [34], [35], where they demonstrate how the new method has reduced the computational complexity. The framework has also been used to develop a convex optimization approach to reduce the fuel consumption in [38]. Also a recent work [43] considered a H-J PDE with viscous term that allowed them to perform a feedback linearisation, which enabled tracking a desired timedependent state on some fixed space point.

In this paper, we derive an explicit solution of a HamiltonJacobi PDE with Hamiltonian corresponding to the triangular flux function. Its solution corresponds to the cumulated vehicle count function in time on a single traffic road. The main contributions of this paper are the following:

- Tracking space- and time-dependent trajectory: we extend the results presented in [39], [42] and present a controller able to drive a state with shocks to any time- and space- dependent vehicle density that also may contain shocks. This is the first boundary controller in the traffic community able to solve such general tasks. Moreover, comparing to [43], our analysis is done for the original LWR system without linearisation and viscosity term.

- The solution of H-J PDE is used to provide conditions on when and which control can be applied: we have considered a general case, when it is not guaranteed that supply at the entrance and demand at the exit are large enough, i.e., it might not always be possible to impose any control. We prove in this work that even in that case the goal will be achieved. For this, we have formulated weak boundary conditions in terms of control restriction functions, see more details in Section III.

This paper is organized as follows. First, we state the problem of finding a boundary control law for a traffic system. In Section II we introduce a relation between LWR and H-J formulations and explicitly calculate the solution of H-J PDE asymptotic in time for the triangular flux function. Then, the problem is stated in H-J formulation. Section III contains the main result (the control laws) and the proof. In Section IV we consider a numerical example verifying our controller. Finally, the concluding remarks are given in Section V.

\section{B. Problem Statement}

The LWR model is based on the conservation of the number of vehicles and can be expressed as

$$
\frac{\partial \rho(x, t)}{\partial t}+\frac{\partial \Phi(\rho(x, t))}{\partial x}=0, \quad \forall(x, t) \in[0, L] \times \mathbb{R}^{+}
$$

where $\rho(x, t):[0, L] \times \mathbb{R}^{+} \rightarrow \mathbb{R}^{+}$indicates the traffic density, and the flux function $\Phi(\rho(x, t)):\left[0, \rho_{\max }\right] \rightarrow \mathbb{R}^{+}$is a Lipschitz continuous and concave function that admits a unique maximum $\phi_{\max }$ (capacity) attained at $\rho_{c}$ (critical density), while $\Phi(0)=\Phi\left(\rho_{\max }\right)=0$ ( $\rho_{\max }$ is the "jam density"). The LWR model is based on an empirically established law [1] that relates density $\rho(x, t)$ and flow $\phi(x, t)$ as $\Phi(\rho(x, t))=\phi(x, t)$, known as a fundamental diagram (FD). We distinguish two density regimes: the traffic has a positive kinematic wave speed if $\rho \in\left[0, \rho_{c}\right]$ (free-flow regime), and the wave speed is negative if $\rho \in\left(\rho_{c}, \rho_{\max }\right]$ (congested regime). Here we use a triangular (bilinear) FD, see Figure 1, proposed by Daganzo [11]:

$$
\Phi(\rho)= \begin{cases}v_{f} \rho, & \rho \in\left[0, \rho_{c}\right] \\ -w\left(\rho-\rho_{\max }\right), & \rho \in\left(\rho_{c}, \rho_{\max }\right]\end{cases}
$$

where $v_{f}$ is the free-flow speed (wave moving forwards), and $-w$ is the congestion speed (wave moving backwards).

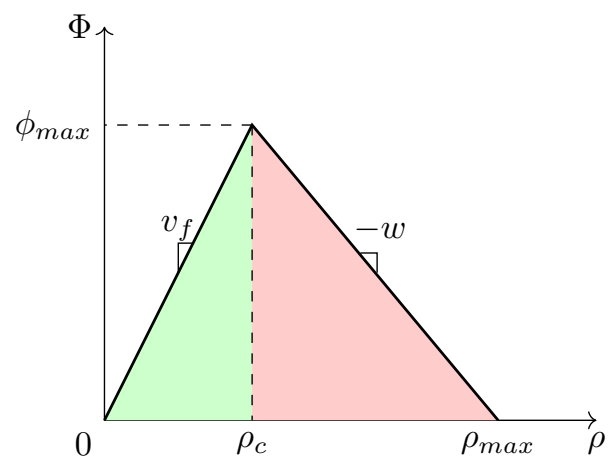

Fig. 1. Triangular Fundamental Diagram: free-flow regime (in green) and congested regime (in red).

Let us fix an initial condition $\rho_{0}(x) \forall x \in[0, L]$, boundary flows $u_{\text {in }}(t)$ and $u_{\text {out }}(t) \forall t \in \mathbb{R}^{+}$(which we treat as control variables), and introduce an initial boundary value problem:

$$
\Sigma=\left\{\begin{array}{l}
\partial_{t} \rho(x, t)+\partial_{x} \Phi(\rho(x, t))=0, \\
\Phi(\rho(0, t))=\phi_{\text {in }}(t), \Phi(\rho(L, t))=\phi_{\text {out }}(t), \\
\rho(x, 0)=\rho_{0}(x) .
\end{array}\right.
$$

Inflows $\phi_{\text {in }}(t)$ and outflows $\phi_{\text {out }}(t)$ are defined $\forall t \in \mathbb{R}^{+}$as

$$
\left\{\begin{array}{l}
\phi_{\text {in }}(t)=\phi_{\text {in }}(u, S)=\min \left\{u_{\text {in }}(t), S(\rho(0, t))\right\}, \\
\phi_{\text {out }}(t)=\phi_{\text {out }}(D, u)=\min \left\{D(\rho(L, t)), u_{\text {out }}(t)\right\},
\end{array}\right.
$$

with $S(\rho(0, t))$ the supply and $D(\rho(L, t))$ the demand functions at the right and left boundary, respectively, given by

$$
\begin{aligned}
& S(\rho)=\min \left\{w\left(\rho_{\max }-\rho\right), v_{f} \rho_{c}\right\}, \\
& D(\rho)=\min \left\{v_{f} \rho, v_{f} \rho_{c}\right\} .
\end{aligned}
$$

Note that the boundary conditions (4) are given in terms of flows. This is equivalent to the weak boundary conditions formulation [31] in terms of densities given in [23]. Thus, the problem given by (3) and (4) is well-posed.

Now we define the desired space-and time-varying density $\rho_{d}(x, t)$ that should be achieved with the help of boundary control. In order to be admissible $\rho_{d}(x, t) \in \mathbb{R}^{+}$must be a weak entropy solution of the following system: 


$$
\Sigma_{d}=\left\{\begin{array}{l}
\frac{\partial \rho_{d}(x, t)}{\partial t}+\frac{\partial \Phi\left(\rho_{d}(x, t)\right)}{\partial x}=0 \\
\Phi\left(\rho_{d}(0, t)\right)=\phi_{i n_{d}}(t), \Phi\left(\rho_{d}(L, t)\right)=\phi_{\text {out }_{d}}(t) \\
\rho_{d}(x, 0)=\rho_{0_{d}}(x)
\end{array}\right.
$$

where inflows and outflows in the desired system must satisfy $\phi_{i n_{d}}(t) \leq S\left(\rho_{d}(0, t)\right)$ and $\phi_{\text {out }_{d}}(t) \leq D\left(\rho_{d}(L, t)\right)$.

Thus, in scope of this paper, our objective is to find $\forall t \in \mathbb{R}^{+}$ boundary control laws $u_{\text {in }}(t)$ and $u_{\text {out }}(t)$ such that the density follows a desired trajectory $\rho_{d}(x, t)$ as $t \rightarrow \infty$.

Finally, throughout this paper we assume the following:

Assumption 1. The initial conditions have left the system, thus, the solution of $\Sigma$ is determined by the boundaries only.

Assumption 2. There exists $\varepsilon>0$ such that $\phi_{\text {in }}$ and $\phi_{\text {out }}$ from $\Sigma$ satisfy the following inequalities in its time average:

$$
\begin{gathered}
\int_{t}^{t+T} \phi_{\text {in }}(\tau) d \tau \leq T \phi_{\text {max }}-\varepsilon \text { and } \int_{t}^{t+T} \phi_{\text {out }}(\tau) d \tau \leq T \phi_{\max }-\varepsilon, \\
\text { where } t>0 \text { and } T=\min \left\{\frac{L}{v_{f}}, \frac{L}{\omega}\right\} .
\end{gathered}
$$

Thus, flows can not hold their maximal values during the time interval given by $T$. This assumption is needed to prove exponential convergence to a desired profile, see details in the proof of Theorem 1.

Remark 1. Note that if Assumption 2 is satisfied, then by taking $t \geq t_{\text {min }}$, where $t_{\text {min }}$ is defined as

$$
t_{\min }=\min \left\{\frac{L}{v_{f}}, \frac{L}{w}\right\}\left(1+\left\lceil\frac{1}{\varepsilon} \int_{0}^{L}\left(\rho_{\max }+\rho_{c}\right)\right\rceil\right),
$$

Assumption 1 holds trivially, as it is shown in Appendix II.

\section{Preliminaries}

As mentioned above, discontinuities may emerge in solutions to conservation laws in finite time even for smooth initial conditions. Note that control enters the system through the minimum function (4). If density would be in one regime only, then it would be possible to reduce (4) to $\phi_{i n}(t)=u_{i n}(t)$ and $\phi_{\text {out }}(t)=u_{\text {out }}(t)$. However, to solve control problems for a traffic state in a mixed regime, we must handle (4). This might be problematic for an LWR PDE system due to the necessity to track shock positions.

Thus, we will equivalently use the Hamilton-Jacobi formulation, which gives a closed-form solution corresponding to a minimum function, and which allows to analyse weak boundary conditions explicitly. In particular, we will be able to analyse when and for how long prescribed control values are accepted by the system. Being an integral form of LWR, the solution of Hamilton-Jacobi PDE has no shocks.

\section{A. Link Between H-J and LWR Formulation}

We introduce the cumulative vehicle count also called the Moskowitz function $M(x, t)$ [5], [10]. It should be interpreted as a cumulated (counted) number of vehicles at location $x$ and at time $t$. The density $\rho(x, t)$ and the flow $\Phi(\rho(x, t))$ can be computed from $M(x, t)$ as

$$
\rho(x, t)=-\frac{\partial M(x, t)}{\partial x}, \quad \Phi(\rho(x, t))=\frac{\partial M(x, t)}{\partial t} .
$$

Intuitively, one can see a relation between $\mathrm{HJ}$ and LWR by performing a formal computation, namely by taking the derivative of the density w.r.t. time and the derivative of the flow w.r.t. space (assuming both of them being continuous) and by using (8), and thus obtaining the LWR PDE:

$\frac{\partial \rho(x, t)}{\partial t}+\frac{\partial \Phi(\rho(x, t))}{\partial x}=0 \Leftrightarrow \frac{\partial^{2} M(x, t)}{\partial x \partial t}-\frac{\partial^{2} M(x, t)}{\partial t \partial x}=0$.

The rigorous relation was shown in [8]. Further, we can rewrite $\Phi(\rho(x, t))=\phi(x, t)$ using $(8)$ as

$$
\frac{\partial M(x, t)}{\partial t}-\Phi\left(-\frac{\partial M(x, t)}{\partial x}\right)=0
$$

which is a Hamilton-Jacobi PDE, and $\Phi$ plays the role of a Hamiltonian. Its solution corresponds to the cumulative vehicle count $M(x, t)$. Note that the LWR PDE can be obtained if (9) is differentiated w.r.t. space and expressed in terms of density.

Note that (9) depends only on derivatives of $M(x, t)$. Therefore, for any solution $M(x, t)$ adding any constant $M_{0}$ gives also a solution $M(x, t)+M_{0}$. This is obvious, since we can start numeration of cars from any particular number.

The existence of $M(x, t)$ itself guarantees the conservation of vehicle numbers. A shock corresponds to a discontinuity in the first derivative of $M(x, t)$, then the conservation equations are still valid if $M(x, t)$ is continuous across the shock path.

\section{B. General Solution of $\mathrm{H}-J$}

The H-J PDE (9) can be solved analytically using initial condition function $M_{\mathrm{Ini}}(x)$, upstream $M_{\mathrm{Up}}(t)$ and downstream $M_{\text {Down }}(t)$ boundary condition functions. Note that the boundary conditions should be consistent with the weak boundary conditions formulation (3)-(4).

Thus, let us introduce the following Cauchy problem for the HJ PDE $\forall(x, t) \in[0, L] \times \mathbb{R}^{+}$:

$$
\left\{\begin{array}{l}
\frac{\partial M(x, t)}{\partial t}-\Phi\left(-\frac{\partial M(x, t)}{\partial x}\right)=0 \\
M(x, 0)=M_{\mathrm{Ini}}(x) \\
M(0, t)=M_{\mathrm{Up}}(t) \\
M(L, t)=M_{\text {Down }}(t)
\end{array}\right.
$$

The relation of $M_{\mathrm{Up}}(t)$ and $M_{\text {Down }}(t)$ with $\phi_{\text {in }}(t)$ and $\phi_{\text {out }}(t)$ are studied in detail in Appendix A.

For convenience, let us introduce the value condition function $c(x, t): \operatorname{Dom}(c) \rightarrow \mathbb{R}^{+}$, where $\operatorname{Dom}(c)=$ $\left(\{0, L\} \times \mathbb{R}^{+}\right) \cup((0, L) \times\{0\})$, which aggregates the initial and boundary conditions of (10) (see [28]):

$$
c(x, t)=\left\{\begin{array}{l}
M_{\mathrm{Ini}}(x) \quad t_{s}=0, \\
M_{\mathrm{Up}}(t) \quad x_{s}=0, \\
M_{\text {Down }}(t) \quad x_{s}=L,
\end{array}\right.
$$

where $x_{s}$ and $t_{s}$ are space and time points in space and time, respectively, related to the start of travelling. 
To obtain a closed-form solution of (10), one should treat (10) as a constrained optimization problem [19]. Let us introduce a Legendre-Fenchel transform of the flow $\Phi(\rho)$ as $L(v)$ :

$$
\forall v \in\left[-w, v_{f}\right], \quad L(v)=\sup _{\rho \in\left[0, \rho_{\max }\right]}(\Phi(\rho)-v \rho) .
$$

Let us also introduce $T=t-t_{s}$. The closed-form solution to (10) is known as Lax-Hopf formula, which reads

$$
\begin{aligned}
M(x, t)= & \inf \{c(x-T v, t-T)+T L(v)\} \\
& \text { s.t. } \quad(v, T) \in\left[-w, v_{f}\right] \times \mathbb{R}^{+} \\
& \text {and } \quad\left(x-T v, t_{s}\right) \in \operatorname{Dom}(c) .
\end{aligned}
$$

Thus, $M(x, t)$ is determined by the infimum of the infinite number of functions of the value condition (see also [24]).

\section{Explicit Solution for $\mathrm{t} \rightarrow \infty$}

Our aim here is to obtain an explicit solution of (10) for a triangular FD (2) using (13). The calculations are presented in Appendix A. The final solution $M(x, t)$ is obtained for large enough time, which equivalently means that the effect of initial conditions should have left the system. If Assumptions 1 and 2 hold, then the time can be estimated as in Appendix B:

$$
\begin{aligned}
& \forall(x, t) \in[0, L] \times\left[t_{\text {min }},+\infty\right): \\
& M(x, t)=\min \left\{\int_{0}^{t-\frac{x}{v_{f}}} \phi_{\text {in }}(\tau) d \tau+\int_{0}^{L} \rho_{0}(s) d s,\right. \\
& \left.\int_{0}^{t-\frac{L-x}{w}} \phi_{\text {out }}(\tau) d \tau+\rho_{\max }(L-x)\right\} .
\end{aligned}
$$

\section{Problem Statement in H-J Formulation} 14):

$$
\begin{aligned}
& \forall(x, t) \in[0, L] \times\left[t_{\text {min }},+\infty\right): \\
& M_{d}(x, t)=\min \left\{\int_{0}^{t-\frac{x}{v_{f}}} \phi_{\text {in }}(\tau) d \tau+\int_{0}^{L} \rho_{d_{0}}(s) d s,\right. \\
& t-\frac{L-x}{w} \\
& \left.\int_{0}^{w} \phi_{\text {out }_{d}}(\tau) d \tau+\rho_{\max }(L-x)\right\} .
\end{aligned}
$$

Note that both $M(x, t)$ and $M_{d}(x, t)$ are defined up to a constant since the starting point for the numeration of cars can be arbitrary. Therefore, we will state our problem in HamiltonJacobi formulation as a pointwise convergence of Moskowitz functions $M(x, t)$ to $M_{d}(x, t)$ as $t \rightarrow \infty$.

Problem 1. Given a desired state $\rho_{d}(x, t)$ solving $\Sigma_{d}$, find control laws $u_{i n}(t)$ and $u_{\text {out }}(t)$ for system $\Sigma$ such that

$$
\exists M_{0} \in \mathbb{R}: \forall x \in[0, L] \quad \lim _{t \rightarrow \infty}\left(M(x, t)-M_{d}(x, t)\right)=M_{0} .
$$

Remark 2. Physically, we need to control inflow and outflow such that all the "excess" cars (given by the integral difference in $\rho_{0}(x)$ and $\left.\rho_{d_{0}}(x)\right)$ leave the domain, and then inflows and outflows match the desired ones. When the goal is achieved, the following conditions hold for $\forall t \geq t_{\min }$ :

(i) $\phi_{\text {in }}(t)=\phi_{\text {in }_{d}}(t), \quad($ ii $) \phi_{\text {out }}(t)=\phi_{\text {out }_{d}}(t)$,

$$
\begin{aligned}
& \int_{0}^{t-\frac{x}{v_{f}}}\left(\phi_{\text {in }}(\tau)-\phi_{\text {in }}(\tau)\right) d \tau+ \\
& \int_{0}^{L}\left(\rho_{0}(s)-\rho_{d_{0}}(s)\right) d s=\int_{0}^{t-\frac{L-x}{w}}\left(\phi_{\text {out }}(\tau)-\phi_{\text {out }_{d}}(\tau)\right) d \tau .
\end{aligned}
$$

The derivation of these conditions is given in Appendix III.

\section{CONTROL LAW DESIGN}

Theorem 1. Assume system $\Sigma$ for which Assumptions 1 and 2 hold with the MF solution given by (14), and system $\Sigma_{d}$ for which Assumption 1 holds with the MF solution (15). Then a control law that achieves the goal stated in Problem 1 is given by

$$
\begin{gathered}
\text { (1) } u_{\text {in }}(t)=\phi_{\text {ind }_{d}}(t)-k e(t), \\
\text { (2) } u_{\text {out }}(t)=\phi_{\text {out }_{d}}(t)+k e(t), \quad t \in \mathbb{R}^{+} \\
\text {where } e(t)=\int_{0}^{L}\left(\rho(s, t)-\rho_{d}(s, t)\right) d s \text { and } k>0 .
\end{gathered}
$$

Proof. This proof consists of five subsections: it is shown that $e(t)$ goes to zero as $t \rightarrow \infty$ in subsections 1$)-3$ ), and then we show that this is enough for the convergence of Moskowitz functions in subsections 4) and 5).

Error $e(t)$ is defined as the difference in the overall number of cars in the real and the desired systems. Using (51) for $M(0, t)$ we can rewrite the definition of error as

$$
e(t)=M(0, t)-M_{d}(0, t)+\int_{0}^{t}\left(\phi_{\text {out }}(\tau)-\phi_{\text {out }}(\tau)\right) d \tau .
$$

Now we can rewrite it using (52) for $M(0, t)$ as

$$
\begin{aligned}
e(t) & =\int_{0}^{t}\left(\phi_{\text {in }}(\tau)-\phi_{\text {out }}(\tau)+\phi_{\text {out }_{d}}(\tau)-\phi_{\text {in }}(\tau)\right) d \tau \\
& +\int_{0}^{L}\left(\rho_{0}(s)-\rho_{0_{d}}(s)\right) d s .
\end{aligned}
$$

Error dynamics are the time derivative of (18)

$$
\dot{e}(t)=\phi_{\text {in }}(t)-\phi_{\text {out }}(t)-\phi_{\text {in }}(t)+\phi_{\text {out }}(t) .
$$

The main difficulty with the control of $\Sigma$ is that $\phi_{i n}$ is not always equal to $u_{i n}$ (and similarly $\phi_{\text {out }}$ ), i.e., in some cases we lose the ability to impose any control $u_{\text {in }}(t)$ or $u_{\text {out }}(t)$ on the boundaries. Let us investigate this problem in more details. 
1) Analysis of Flow Restrictions: By definition (8) inflows and outflows are time derivatives of $M(0, t)$ and $M(L, t)$, respectively. First, we focus on the inflow at the upstream boundary defined as $\phi_{i n}(t)=\partial M(0, t) / \partial t$. Let us express $M(0, t)$ by taking the time integral of $\phi_{i n}(t)$ and get

$$
M(0, t)=\int_{0}^{t} \phi_{i n}(\tau) d \tau+M(0,0)
$$

where $M(0,0)=\int_{0}^{L} \rho_{0}(s) d s$ by $(51)$ for $(x, t)=(0,0)$.

At the same time, by (14) for $x=0$ we obtain

$$
\begin{aligned}
M(0, t)= & \min \left\{\int_{0}^{t} \phi_{\text {in }}(\tau) d \tau+\int_{0}^{L} \rho_{0}(s) d s,\right. \\
& \left.\int_{0}^{t-\frac{L}{\omega}} \phi_{\text {out }}(\tau) d \tau+L \rho_{\max }\right\} .
\end{aligned}
$$

Combining (20) with (21), we obtain a minimum problem:

$$
\begin{aligned}
\int_{0}^{t} \phi_{i n}(\tau) d \tau & +\int_{0}^{L} \rho_{0}(s) d s=\min \left\{\int_{0}^{t} \phi_{i n}(\tau) d \tau\right. \\
& \left.+\int_{0}^{L} \rho_{0}(s) d s, \int_{0}^{t-\frac{L}{\omega}} \phi_{\text {out }}(\tau) d \tau+L \phi_{\max }\right\}
\end{aligned}
$$

From (22) one can see that the following must hold

$$
\int_{0}^{t} \phi_{i n}(\tau) d \tau+\int_{0}^{L} \rho_{0}(s) d s \leq \int_{0}^{t-\frac{L}{\omega}} \phi_{o u t}(\tau) d \tau+L \phi_{\max }
$$

In case of equality in (23), we must provide that the righthand term grows more quickly than the left-hand term. Thus, by taking time derivative we obtain $\phi_{\text {in }}(t) \leq \phi_{\text {out }}\left(t-\frac{L}{w}\right)$.

All this can be combined in the following property. We define a control restriction function for the downstream boundary

$g_{\text {in }}(t)=\int_{0}^{t-\frac{L}{\omega}} \phi_{\text {out }}(\tau) d \tau+L \rho_{\max }-\int_{0}^{t} \phi_{\text {in }}(\tau) d \tau-\int_{0}^{L} \rho_{0}(s) d s$,

which represents the difference between two arguments of the minimum. By (23) we obtain that $g_{i n}(t) \geq 0$ always. Moreover, using the definition of $\phi_{i n}(t)$ given by (4), the condition on inflow restriction can be formulated as:

$$
\begin{aligned}
g_{\text {in }}(t)>0: & \phi_{\text {in }}(t)=u_{\text {in }}(t), \\
g_{\text {in }}(t)=0: & \phi_{\text {in }}(t)=\min \left[u_{\text {in }}(t), \phi_{\text {out }}\left(t-\frac{L}{w}\right)\right] .
\end{aligned}
$$

Note that the notation of control restriction should be understood as saturation control, with $\phi_{\text {out }}\left(t-\frac{L}{w}\right)$ being the saturation time-varying threshold.

Similarly, we proceed with $M(L, t)$ to analyse $\phi_{\text {out }}(t)$ for the downstream boundary, and get its control restriction function:

$$
g_{\text {out }}(t)=\int_{0}^{t-\frac{L}{v_{f}}} \phi_{\text {in }}(\tau) d \tau+\int_{0}^{L} \rho_{0}(s) d s-\int_{0}^{t} \phi_{\text {out }}(\tau) d \tau,
$$

and the following condition on outflow restriction

$$
\begin{aligned}
& g_{\text {out }}(t)>0: \phi_{\text {out }}(t)=u_{\text {out }}(t) \\
& g_{\text {out }}(t)=0: \phi_{\text {out }}(t)=\min \left[u_{\text {out }}(t), \phi_{\text {in }}\left(t-\frac{L}{v_{f}}\right)\right] .
\end{aligned}
$$

Any boundary control can be imposed if $g_{\text {in }}>0$ and $g_{\text {out }}>0$.

Defining $R(t)=\int_{0}^{L} \rho(s, t) d s$ and $R_{0}=R(0)$, and using the equality of (51) and (52) for $x=0$ we obtain $\forall t^{\prime} \in \mathbb{R}^{+}$

$$
R\left(t^{\prime}\right)=R_{0}+\int_{0}^{t^{\prime}} \phi_{\text {in }}(\tau) d \tau-\int_{0}^{t^{\prime}} \phi_{\text {out }}(\tau) d \tau .
$$

Thus, the control restriction functions can be rewritten as

$$
\begin{aligned}
& g_{\text {in }}(t)=L \rho_{\text {max }}-R\left(t^{\prime}\right)-\int_{t-\frac{L}{w}}^{t^{\prime}} \phi_{\text {out }}(\tau) d \tau-\int_{t^{\prime}}^{t} \phi_{\text {in }}(\tau) d \tau, \\
& g_{\text {out }}(t)=R\left(t^{\prime}\right)-\int_{t-\frac{L}{v_{f}}}^{t^{\prime}} \phi_{\text {in }}(\tau) d \tau-\int_{t^{\prime}}^{t} \phi_{\text {out }}(\tau) d \tau .
\end{aligned}
$$

Note also that inflows and outflows are bounded, i.e. $\phi_{\text {in }} \leq$ $\phi_{\max }$ and $\phi_{\text {out }} \leq \phi_{\max }$, where $\phi_{\max }=v_{f} \rho_{c}$. To find the time interval, when no control law can be imposed, we set $g_{i n}(t)=0$ and then express $R\left(t^{\prime}\right)$ from (27):

$$
\begin{gathered}
R\left(t^{\prime}\right)=L \rho_{\max }-\int_{t-\frac{L}{w}}^{t^{\prime}} \phi_{\text {out }}(\tau) d \tau-\int_{t^{\prime}}^{t} \phi_{\text {in }}(\tau) d \tau \geq L \rho_{\max } \\
-\int_{t-\frac{L}{w}}^{t} \phi_{\max } d \tau-\int_{t^{\prime}}^{t^{\prime}} \phi_{\max } d \tau=L \rho_{\max }-\frac{L}{w} \phi_{\max }=L \rho_{c} .
\end{gathered}
$$

The same steps are performed for $g_{\text {out }}(t)=0$, and we get

$$
\begin{gathered}
g_{\text {in }}(t)=0 \quad \Rightarrow \quad R\left(t^{\prime}\right) \geq L \rho_{c} \quad \forall t^{\prime} \in\left[t-\frac{L}{w}, t\right], \\
g_{\text {out }}(t)=0 \quad \Rightarrow \quad R\left(t^{\prime}\right) \leq L \rho_{c} \quad \forall t^{\prime} \in\left[t-\frac{L}{v_{f}}, t\right] .
\end{gathered}
$$

This means that not any control law can be imposed at the inflow boundary only if during the preceding time interval $\left[t-\frac{L}{w}, t\right]$ the mean density was bigger than the critical density (and inversely for the outflow boundary).

By Assumption 2, setting $g_{\text {in }}(t)=0$ in (27) implies $R(t) \geq$ $L \rho_{c}+\varepsilon$ and $R(t-L / w) \geq L \rho_{c}+\varepsilon$, as well as $g_{\text {out }}(t)=0$ implies $R(t) \leq L \rho_{c}-\varepsilon$ and $R\left(t-L / v_{f}\right) \leq L \rho_{c}-\varepsilon$.

Let us consider (28) to investigate whether it is possible that control can not be imposed at both boundaries simultaneously. We pick up some time point $t$ such that $g_{\text {in }}(t)=0$ and some time point $t^{\prime} \in\left[t, t+L / v_{f}\right]$ with $g_{\text {out }}\left(t^{\prime}\right)=0$. As written above, $g_{\text {in }}(t)=0$ implies $R(t) \geq L \rho_{c}+\varepsilon$. However, $t \in$ 
$\left[t^{\prime}-L / v_{f}, t^{\prime}\right]$, thus $R(t) \leq L \rho_{c}$ for $g_{\text {out }}\left(t^{\prime}\right)=0$ by (28). This is a contradiction, since satisfying both $R(t) \geq L \rho_{c}+\varepsilon$ and $R(t) \leq L \rho_{c}$ at the same $t$ is impossible. Thus, the time point $t^{\prime}$ when $g_{\text {out }}\left(t^{\prime}\right)=0$ can occur at least after the interval $L / v_{f}$ after $g_{i n}(t)=0$.

Moreover, if $t^{\prime} \geq t+L / v_{f}$ and $g_{\text {out }}\left(t^{\prime}\right)=0$, then $R\left(t^{\prime}-\right.$ $\left.L / v_{f}\right) \leq L \rho_{c}-\varepsilon$. The maximal inflow is $\phi_{\max }$, therefore the difference in the integral densities $R\left(t^{\prime}-L / v_{f}\right)-R(t)$ can be passed at least in time $t^{\prime}-L / v_{f}-t \geq 2 \varepsilon / \phi_{\max }$. Performing the same analysis for the inverse case, we conclude that

$$
\begin{gathered}
g_{\text {in }}(t)=0 \quad \Rightarrow \quad g_{\text {out }}\left(t^{\prime}\right)>0 \forall t^{\prime} \in\left[t, t+\frac{L}{v_{f}}+\frac{2 \varepsilon}{\phi_{\text {max }}}\right], \\
g_{\text {out }}(t)=0 \quad \Rightarrow \quad g_{\text {in }}\left(t^{\prime}\right)>0 \forall t^{\prime} \in\left[t, t+\frac{L}{w}+\frac{2 \varepsilon}{\phi_{\max }}\right] .
\end{gathered}
$$

Thus, it is impossible for two boundaries to be unable to accept the control simultaneously, and the periods of "uncontrollability" are separated in time by at least $\frac{L}{v_{f}}+\frac{2 \varepsilon}{\phi_{\max }}$ or $\frac{L}{w}+\frac{2 \varepsilon}{\phi_{\max }}$.

2) Dynamics of $\mathrm{e}(\mathrm{t})$ : At each moment either one of control restriction functions or none of them is zero (29). Hence, we separate the dynamics of $e(t)$ into three possible cases.

1. Assume both $g_{\text {in }}(t)>0$ and $g_{\text {out }}(t)>0$. Then $\phi_{i n}(t)=$ $\phi_{i n_{d}}(t)-k e(t)$ and $\phi_{\text {out }}(t)=\phi_{\text {out }}(t)+k e(t)$. According to (19), the error dynamics are given by

$$
\dot{e}(t)=-2 k e(t),
$$

and, thus, $e(t)$ converges exponentially to zero.

2. Assume $g_{i n}(t)=0$. Then $\phi_{i n}(t) \leq \phi_{i n_{d}}(t)-k e(t)$ and $\phi_{\text {out }}(t)=\phi_{\text {out }_{d}}(t)+k e(t)$, which means

$$
\dot{e}(t) \leq-2 k e(t) \text {. }
$$

Thus, $e(t)>0$ implies even faster convergence to zero. If $e(t)<0$, such dynamics can diverge from zero. However, it is possible to show that after a period of not being able to impose any control, the error will not be further away from zero than at the beginning of the period. Consider the desired control restriction function for the upstream boundary $g_{i n_{d}}(t)$ :

$$
\begin{aligned}
g_{\text {ind }}(t) & =L \rho_{\max }-R_{d}\left(t^{\prime}\right)-\int_{t-\frac{L}{w}}^{t^{\prime}} \phi_{\text {out }}(\tau) d \tau \\
& -\int_{t^{\prime}}^{t} \phi_{\text {ind }}(\tau) d \tau \geq 0, \text { for } t^{\prime} \in\left[t-\frac{L}{w}, t\right] .
\end{aligned}
$$

Using $e\left(t^{\prime}\right)=R\left(t^{\prime}\right)-R_{d}\left(t^{\prime}\right)$, we obtain

$$
\begin{aligned}
g_{\text {in }}(t) & -g_{\text {in }}(t)=\int_{t-\frac{L}{w}}^{t^{\prime}}\left(\phi_{\text {out }}(\tau)-\phi_{\text {out }}(\tau)\right) d \tau \\
& +\int_{t^{\prime}}^{t}\left(\phi_{\text {in }}(\tau)-\phi_{\text {in }}(\tau)\right) d \tau+e\left(t^{\prime}\right) \geq 0 .
\end{aligned}
$$

Using the properties $\phi_{i n}(t) \leq \phi_{i n_{d}}(t)-k e(t)$ and $\phi_{\text {out }}(t)=$ $\phi_{\text {out }_{d}}(t)+k e(t)$, we obtain

$$
e\left(t^{\prime}\right)+k \int_{t-\frac{L}{w}}^{t^{\prime}} e(\tau) d \tau-k \int_{t^{\prime}}^{t} e(\tau) d \tau \geq 0 .
$$

We substitute $t^{\prime}=t$ to get the first inequality, and then $t^{\prime}=$ $t-\frac{L}{w}$ to get the second one. The sum of these inequalities yields

$$
e(t)+e\left(t-\frac{L}{w}\right) \geq 0 .
$$

3. Assume $g_{\text {out }}(t)=0$. Then $\phi_{\text {out }}(t) \leq \phi_{\text {out }}(t)+k e(t)$ and $\phi_{i n}(t)=\phi_{i n_{d}}(t)-k e(t)$, which yields

$$
\dot{e}(t) \geq-2 k e(t) \text {. }
$$

Using the same analysis as above for $e(t)>0$, we obtain

$$
e(t)+e\left(t-\frac{L}{v_{f}}\right) \leq 0 .
$$

3) Proof that $\mathrm{e}(\mathrm{t})$ converges to zero: In the table below we have summarized three regimes of error dynamics:

\begin{tabular}{|l|l|l|}
\hline \multicolumn{3}{|c|}{ Summary of error regimes } \\
\hline Regime 1 & $g_{\text {in }}(t)>0$, & $\dot{e}(t)=-2 k e(t)$ \\
& $g_{\text {out }}(t)>0$ & \\
\hline Regime 2 & $g_{\text {in }}(t)=0$, & $\dot{e}(t) \leq-2 k e(t)$ \\
& $g_{\text {out }}\left(t^{\prime}\right)>0$ & \\
& $\forall t^{\prime} \in\left[t, t+\frac{L}{v_{f}}+\frac{2 \varepsilon}{\phi_{\max }}\right]$ & \\
\hline Regime 3 & $g_{\text {in }}(t)>0$, & $\dot{e}(t) \geq-2 k e(t)$ \\
& $g_{\text {out }}\left(t^{\prime}\right)=0$ & \\
& $\forall t^{\prime} \in\left[t, t+\frac{L}{w}+\frac{2 \varepsilon}{\phi_{\max }}\right]$ & \\
\hline
\end{tabular}

The regimes can alternate as depicted in Figure 2. In this part of the proof we will show that only positive error can enter regime 2 at some time, while it can enter regime 3 only being negative. Thus, if the error is positive in regime 1 (green circle), it either remains in regime 1 forever, where the exponential convergence to zero is guaranteed by (30), or it enters regime 2 (blue circle). Then, being positive, by (31) the error converges to zero even faster than in regime 1. However, it can also become negative, and in this case the error might diverge from zero. Nevertheless, the divergence from zero can last only for a bounded time interval, and the absolute value of the error term can not exceed its value some time ago (see (33)). As this happens, the error enters again regime 1 as a negative term. It either stays there forever, or switches to regime 3, where it goes to zero even more quickly by (34). The rest can be described in a symmetric manner. Recall also that regimes 2 and 3 are always separated by at least $\frac{L}{v_{f}}+\frac{2 \varepsilon}{\phi_{\max }}$ or $\frac{L}{w}+\frac{2 \varepsilon}{\phi_{\max }}$. Further, we provide a strict proof of the exponential convergence of the error term to zero.

Imagine a time axis split into three types of intervals corresponding to three different regimes as shown in Figure 3. Recalling that regimes 2 and 3 cannot occur in a row, we can observe, e.g., a sequence like this: $12121313121 \ldots$. Thus, it is possible that after regime 2 and then regime 1 , the regime 2 comes again, since nothing prohibits $g_{i n}(t)$ to become zero again almost immediately. We denote this sequence of regimes as " $g_{i n}(t)=0$ sometimes" which is defined as the largest row of regimes 1 and 2 that starts and ends with regime 2 and does not contain any interval of regime 3 . The same we can do with the regime sequence containing regimes 1 and 3 and call it " $g_{\text {out }}(t)=0$ sometimes". These sequences " $g_{\text {in }}(t)=0$ 


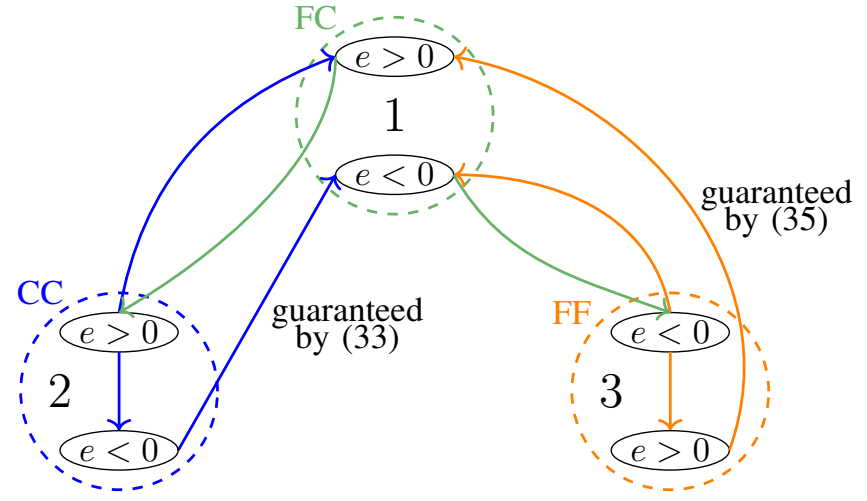

Fig. 2. Diagram of regimes illustrating how they can alternate. Arrows denote possible regime switches. FC, CC and FF are used to denote regimes on the boundaries, where $\mathrm{F}$ stays for free-flow and $\mathrm{C}$ for congested regime.

$e(t)$

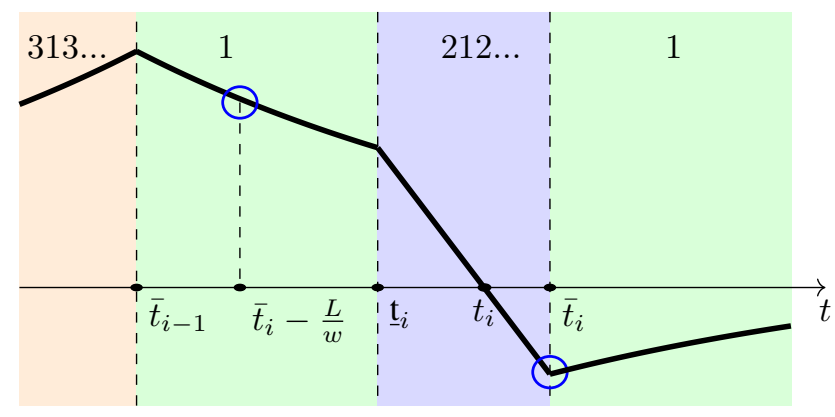

Fig. 3. A possible error behaviour $e(t)$ (thick black line). From left to right: divergence for " $g_{\text {out }}(\boldsymbol{t})=\mathbf{0}$ sometimes" (in orange); exponential convergence in regime $\mathbf{1}$ (in green); fast convergence for " $g_{\text {in }}(t)=\mathbf{0}$ sometimes" (in blue); then divergence for $e\left(\boldsymbol{t}^{\prime}\right)<\mathbf{0} \forall \boldsymbol{t}^{\prime} \in\left[\boldsymbol{t}_{\boldsymbol{i}}, \overline{\boldsymbol{t}}_{\boldsymbol{i}}\right]$; exponential convergence in regime 1 . Blue empty circles are related to (37).

sometimes" and "gout $(t)=0$ sometimes" alternate strictly, always having regime 1 between them. Finally, for a time interval corresponding to the regime (or regime sequence) with index $i$ we can define entrance time $\underline{\mathrm{t}}_{i}$ and exit time $\bar{t}_{i}$. By (29) we see that $\underline{\mathrm{t}}_{i}-\bar{t}_{i-1} \geq \frac{L}{\omega}+\frac{2 \varepsilon}{\phi_{\max }}$ if the regime $i$ is $" g_{\text {in }}(t)=0$ sometimes", and $\underline{\mathrm{t}}_{i}-\bar{t}_{i-1} \geq \frac{L}{v_{f}}+\frac{2 \varepsilon}{\phi_{\max }}$ if the regime $i$ is " $g_{\text {out }}(t)=0$ sometimes".

Let us fix $i$ corresponding to " $g_{i n}(t)=0$ sometimes" (the other case is symmetric). First of all, by (33) we obtain

$$
g_{i n}\left(\underline{\mathbf{t}}_{i}\right)=0 \quad \text { and } \quad g_{\text {in }}\left(\bar{t}_{i}\right)=0 .
$$

Therefore

$$
e\left(\underline{\mathbf{t}}_{i}\right)+e\left(\underline{\mathrm{t}}_{i}-\frac{L}{w}\right) \geq 0
$$

and

$$
e\left(\bar{t}_{i}\right)+e\left(\bar{t}_{i}-\frac{L}{w}\right) \geq 0 .
$$

It is clear that $\mathrm{t}_{i}-\frac{L}{w} \geq \bar{t}_{i-1}$, which means that the dynamics of $e$ in the interval $\left[\underline{\mathrm{t}}_{i}-\frac{L}{w}, \mathrm{t}_{i}\right]$ are exponential. Thus, both $e\left(\underline{\mathbf{t}}_{i}\right)$ and $e\left(\underline{\mathrm{t}}_{i}-\frac{L}{w}\right)$ have the same sign, and by (36) they are both positive. A similar analysis can be done for the regime sequence " $g_{\text {out }}(t)=0$ sometimes", which means that from $g_{\text {out }}(t)=0$ to $g_{\text {in }}(t)=0$ the error term is positive and from $g_{\text {in }}(t)=0$ to $g_{\text {out }}(t)=0$ the error term is negative (and thus $e\left(\bar{t}_{i}\right)<0$ ). Consequently, inside each regime sequence $i$ there should be a time point $t_{i}$, when $e\left(t_{i}\right)=0$.

Now, by (37) and using that $e\left(\bar{t}_{i}\right)$ is negative, we see that $e\left(\bar{t}_{i}-\frac{L}{w}\right) \geq 0$, which means that $\bar{t}_{i}-\frac{L}{w} \leq t_{i}$ (see Figure 3 ).

On the time interval $\left[\bar{t}_{i-1}, \underline{\mathrm{t}}_{i}\right]$ it is clear that the convergence is exponential (regime 1). On the time interval $\left[\underline{\mathrm{t}}_{i}, t_{i}\right]$ the dynamics are $\dot{e} \leq-2 k e$, and $e(t) \geq 0$. Time point $\bar{t}_{i}-\frac{L}{w} \in$ $\left[\bar{t}_{i-1}, t_{i}\right]$, therefore

$$
e\left(\bar{t}_{i}-\frac{L}{w}\right) \leq e\left(\bar{t}_{i-1}\right) \exp ^{-2 k\left(\bar{t}_{i}-\frac{L}{w}-\bar{t}_{i-1}\right)} .
$$

We can write the same for the absolute values,

$$
\left|e\left(\bar{t}_{i}-\frac{L}{w}\right)\right| \leq\left|e\left(\bar{t}_{i-1}\right)\right| \exp ^{-2 k\left(\bar{t}_{i}-\frac{L}{w}-\bar{t}_{i-1}\right)} .
$$

Now from (37) and the fact that $e\left(\bar{t}_{i}\right)<0$ we see that $\left|e\left(\bar{t}_{i}\right)\right| \leq$ $\left|e\left(\bar{t}_{i}-\frac{L}{w}\right)\right|$, thus

$$
\left|e\left(\bar{t}_{i}\right)\right| \leqslant\left|e\left(\bar{t}_{i-1}\right)\right| \exp ^{-2 k\left(\bar{t}_{i}-\frac{L}{w}-\bar{t}_{i-1}\right)} .
$$

We can write $\bar{t}_{i}-\bar{t}_{i-1} \geq \underline{\mathrm{t}}_{i}-\bar{t}_{i-1} \geq \frac{L}{\omega}+\frac{2 \varepsilon}{\phi_{\max }}$, which yields

$$
\begin{aligned}
\frac{\bar{t}_{i}-\frac{L}{w}-\bar{t}_{i-1}}{\bar{t}_{i}-\bar{t}_{i-1}} & =1-\frac{\frac{L}{w}}{\bar{t}_{i}-\bar{t}_{i-1}} \geq 1-\frac{\frac{L}{w}}{\frac{L}{\omega}+\frac{2 \varepsilon}{\phi_{\max }}} \\
& =\frac{\frac{2 \varepsilon}{\phi_{\max }}}{\frac{L}{\omega}+\frac{2 \varepsilon}{\phi_{\max }}}=\frac{2 \varepsilon \omega}{L \phi_{\max }+2 \varepsilon \omega} .
\end{aligned}
$$

The time interval in the exponential function can be bounded

$$
\bar{t}_{i}-\frac{L}{w}-\bar{t}_{i-1} \geq\left(\frac{2 \varepsilon \omega}{L \phi_{\max }+2 \varepsilon \omega}\right)\left(\bar{t}_{i}-\bar{t}_{i-1}\right),
$$

which finally leads to

$$
\left|e\left(\bar{t}_{i}\right)\right| \leq\left|e\left(\bar{t}_{i-1}\right)\right| \exp ^{-2 k\left(\frac{2 \varepsilon \omega}{L \phi_{\max }+2 \varepsilon \omega}\right)\left(\bar{t}_{i}-\bar{t}_{i-1}\right)},
$$

which proves the exponential convergence to zero of $e(t)$.

4) Proof of Integral Inflow Convergence: To proceed we need to show that the integral of difference of inflows in the real and in the desired system has a limit, as it is required for the convergence of the Moskowitz functions as stated in Problem 1 (see next subsection, where the existence of this limit is used for the introduction of constant $M_{0}$ ):

$$
\exists \lim _{t \rightarrow \infty} \int_{0}^{t}\left(\phi_{i n}(\tau)-\phi_{i n_{d}}(\tau)\right) d \tau .
$$

By the Cauchy criterion for the convergence of functions, it suffices to show that

$$
\lim _{t_{1}, t_{2} \rightarrow \infty} \int_{t_{1}}^{t_{2}}\left(\phi_{i n}(\tau)-\phi_{i n_{d}}(\tau)\right) d \tau=0
$$

for any $t_{1}$ and $t_{2}$, where $t_{2}>t_{1}$. First, we find an upper bound for this limit. By combining (24) with (17) we obtain $\phi_{i n} \leq \phi_{i n_{d}}-k e(t)$, thus, we can write $\forall t_{1}, t_{2} \rightarrow \infty$

$$
\int_{t_{1}}^{t_{2}}\left(\phi_{i n}(\tau)-\phi_{i n_{d}}(\tau)\right) d \tau \leq-k \int_{t_{1}}^{t_{2}} e(\tau) d \tau \rightarrow 0,
$$


which implies

$$
\lim _{t_{1}, t_{2} \rightarrow \infty} \int_{t_{1}}^{t_{2}}\left(\phi_{i n}(\tau)-\phi_{i n_{d}}(\tau)\right) d \tau \leq 0 .
$$

Now let us estimate the lower bound for the limit (40). Thereby, we distinguish two possible cases:

1) Assume $g_{i n}(t)>0$. In this case:

$$
\int_{t-\frac{L}{w}}^{t}\left(\phi_{i n}(\tau)-\phi_{i n_{d}}(\tau)\right) d \tau \geq-k \int_{t-\frac{L}{w}}^{t}|e(\tau)| d \tau .
$$

2) Assume $g_{i n}(t)=0$. Using (32) for $t^{\prime}=t-\frac{L}{w}$, we get

$$
\int_{t-\frac{L}{w}}^{t}\left(\phi_{i n}(\tau)-\phi_{i n_{d}}(\tau)\right) d \tau \geq-e\left(t-\frac{L}{w}\right) \geq-\left|e\left(t-\frac{L}{w}\right)\right| .
$$

The combination of two latter expression yields

$$
\int_{t-\frac{L}{w}}^{t}\left(\phi_{i n}(\tau)-\phi_{i n_{d}}(\tau)\right) d \tau \geq-k \int_{t-\frac{L}{w}}^{t}|e(\tau)| d \tau-\left|e\left(t-\frac{L}{w}\right)\right|
$$

Now let us divide the time interval $\left[t_{1}, t_{2}\right]$ into subintervals of length $L / w$. Thus, (42) can be rewritten for a larger time interval as

$$
\begin{aligned}
\int_{t_{1}}^{t_{2}}\left(\phi_{i n}(\tau)-\phi_{i n_{d}}(\tau)\right) d \tau & \geq-k \int_{t_{1}}^{t_{2}}|e(\tau)| d \tau \\
& -\sum_{n=0}^{\left\lfloor\frac{t_{2}-t_{1}}{L / w}\right\rfloor}\left|e\left(t_{1}+n \frac{L}{w}\right)\right| .
\end{aligned}
$$

where the sum goes over intervals of size $L / w$, i.e., $t_{1}, t_{1}+$ $L / w, \ldots, t_{2}$. If we take the time limit of the latter expression for $t_{1}, t_{2} \rightarrow \infty$, both right-hand terms converge to zero, as for the sum term we can apply the integral test for convergence:

$$
\lim _{t_{1}, t_{2} \rightarrow \infty} \int_{t_{1}}^{t_{2}}\left(\phi_{i n}(\tau)-\phi_{i n_{d}}(\tau)\right) d \tau \geq 0 .
$$

The combination of (43) and (41) provides that the limit is zero, which proves the existence of the limit of the difference integral between inflows in the real and desired systems.

5) Proof of Convergence of Moskowitz Functions: Finally, let us define two auxiliary Moskowitz functions

$$
\begin{gathered}
M_{1}(x, t)=R_{0}-R_{0_{d}}+\int_{0}^{t-\frac{x}{v_{f}}}\left(\phi_{\text {in }}(\tau)-\phi_{\text {ind }}(\tau)\right) d \tau, \\
M_{2}(x, t)=\int_{0}^{t-\frac{L-x}{w}}\left(\phi_{\text {out }}(\tau)-\phi_{\text {out }_{d}}(\tau)\right) d \tau .
\end{gathered}
$$

Notice that $M_{1}(x, t)$ and $M_{2}(x, t)$ correspond to the left and to the right side of (16) (iii), which must hold for large $t$. First of all, using $e_{0}=R_{0}-R_{0_{d}}$ and the dynamics of $e(t)$ given by (19), we obtain that $\forall x \in[0, L]$

$$
\begin{aligned}
\lim _{t \rightarrow \infty} & \left(M_{1}(x, t)-M_{2}(x, t)\right)= \\
& \int_{0}^{\infty}\left(\phi_{\text {in }}(\tau)-\phi_{\text {in }}(\tau)-\phi_{\text {out }}(\tau)+\phi_{\text {out }_{d}}(\tau)\right) d \tau+e_{0} \\
\quad= & \lim _{t \rightarrow \infty} e(t)=0 .
\end{aligned}
$$

Moreover, as it was proven in the previous subsection (39), $M_{1}(x, t)$ has a limit. Therefore, we can define

$$
M_{0}:=\lim _{t \rightarrow \infty} M_{1}(x, t),
$$

and by (46) we get

$$
\lim _{t \rightarrow \infty} M_{2}(x, t)=M_{0} .
$$

We can also define the error terms as $\tilde{M}_{1}(x, t)=M_{1}(x, t)-$ $M_{0}$ and $\tilde{M}_{2}(x, t)=M_{2}(x, t)-M_{0}$.

Finally, recall that the desired Moskowitz function given by (15) can be expressed as

$$
M_{d}(x, t)=\min \left\{M_{\mathrm{Up}_{d}}(x, t), M_{\text {Down }_{d}}(x, t)\right\},
$$

and using (14), (44) and (45) we obtain

$M(x, t)=\min \left\{M_{\mathrm{Up}_{d}}(x, t)+M_{1}(x, t), M_{\text {Down }_{d}}(x, t)+M_{2}(x, t)\right\}$,

or

$$
\begin{aligned}
M(x, t)= & \min \left\{M_{\mathrm{Up}_{d}}(x, t)+\bar{M}_{1}(x, t),\right. \\
& \left.M_{\text {Down }_{d}}(x, t)+\bar{M}_{2}(x, t)\right\}+M_{0} .
\end{aligned}
$$

Minimum is a continuous function on both arguments, thus we obtain for $t \rightarrow \infty$ that:

$$
M(x, t) \rightarrow M_{d}(x, t)+M_{0} \quad \forall x \in[0, L],
$$

as stated in Problem 1.

After we have solved the problem stated in Hamilton-Jacobi formulation, let us establish the link to LWR formulation.

Lemma 1. Problem 1 is equivalent to the integral convergence of densities over arbitrarily small intervals, i.e., $\forall a, b$ : $0 \leq a<$ $b \leq L$ we obtain

$$
\lim _{t \rightarrow \infty} \int_{a}^{b}\left(\rho(s, t)-\rho_{d}(s, t)\right) d s=0,
$$

where $a$ and $b$ can be arbitrarily close points in space.

Proof. By (51) we can write

$$
M(a, t)-M(b, t)=\int_{a}^{L} \rho(s, t) d s-\int_{b}^{L} \rho(s, t) d s=\int_{a}^{b} \rho(s, t) d s,
$$

and

$$
M_{d}(a, t)-M_{d}(b, t)=\int_{a}^{b} \rho_{d}(s, t) d s .
$$

For $x=a$ and $x=b$ in Problem 1 we get $M(a, t) \rightarrow$ $M_{d}(a, t)+M_{0}, M(b, t) \rightarrow M_{d}(b, t)+M_{0}$. This is equivalent 
to $M(a, t)-M(b, t) \rightarrow M_{d}(a, t)-M_{d}(b, t)$, which by (48) and (49) we rewrite as

$$
\int_{a}^{b} \rho(s, t) d s \rightarrow \int_{a}^{b} \rho_{d}(s, t) d s .
$$

Remark 3. Notice that pointwise convergence of two functions does not imply the convergence for their derivatives in any of $L^{p}$ norms. However, we reach the equality of densities over arbitrarily small intervals, which means that the state approximates the desired trajectory as time goes to infinity.

Remark 4. Note that Assumption 2 is non-limiting. Indeed, it requires that the flow integral over time $\mathrm{T}$ is less than except when vehicles enter and leave the system at maximum rate during T. Obviously, in this case, it is also possible to reach the goal. The need to use Assumption 2 comes from the fact that at maximum flow by (28) both $g_{i n}(t)=0$ and $g_{\text {out }}(t)=0$ for the same $t$, which means that with the slightest fluctuation a boundary becomes "uncontrollable". Thus, the state with maximum flows at both boundaries during time $\mathrm{T}$ is "unstable", and therefore it is impossible to prove the exponential convergence of the error term $e(t)$.

\section{A. Numerical Scheme}

We illustrate the efficiency of feedback control terms (17) by driving a state being inially almost completely in a traffic jam to a desired space- and time-dependent vehicle density being in a mixed regime. The numerical scheme we use is a standard Godunov scheme [4] with a space interval $[0, L]$ divided into $n=500$ cells, i.e. $\Delta x=L / n$, and a time discretization step $\Delta t$ chosen such that the Courant-FriedrichLewy condition is satisfied [6]. This condition is necessary to prevent the violation of information flow.

The integral corresponding to the feedback term is computed using the Riemann summation over cells $i \in\{2 . . n-1\}$. Recall that in general the minimum is not always resolved to the benefit of the control variable. In this section we demonstrate the efficiency of using a state feedback for a road that is initially empty at the entry and in the traffic jam at the exit. Thus, both boundaries accept controls, i.e. $\varphi_{\text {in }}(t)=u_{\text {in }}(t)$ and $\varphi_{\text {out }}(t)=u_{\text {out }}(t)$.

For the simulation we set the following parameters (which are taken from the real traffic data [36]):

$$
\begin{aligned}
& v_{f}=16.67 \mathrm{~m} / \mathrm{s}, w=7.14 \mathrm{~m} / \mathrm{s}, \rho_{\max }=0.181, \mathrm{veh} / \mathrm{m}, \\
& \rho_{c}=0.054 \mathrm{veh} / \mathrm{m}, L=1000 \mathrm{~m},
\end{aligned}
$$

and for initial time the system is given as

$\rho_{0}(0 \leq x<1 / 4 L)=0, \quad$ and $\quad \rho_{0}(1 / 4 L \leq x \leq L)=\rho_{\max }$.

Thus, we will consider the system being almost completely in the traffic jam as initial condition.

As a target state we consider a vehicle density in a mixed case regime, whose evolution is given by "ghost" cells (which are set by copying the value from the neighbour cell) with its maximum value by at least $\varepsilon$, which is always possible,

The boundary values are prescribed in terms of densities, which are transformed into flows by using the supply-demand formulation (5). We will demonstrate, how the feedback term given by $-k e(t)$ for the upstream and $+k e(t)$ for the downstream boundary improves the result and provides the asymptotic convergence targeting the desired profile. Two control strategies are compared:

1) No feedback is performed, i.e. only $u_{i n}(t)=\phi_{i n_{d}}(t)$ and $u_{\text {out }}(t)=\phi_{\text {out }_{d}}(t)$.

2) Feedback terms $u_{\text {in }}(t)=\phi_{i n_{d}}(t)-k e(t), u_{\text {out }}(t)=$ $\phi_{\text {out }_{d}}(t)+k e(t)$ are applied.

\section{B. Results}

In Figure 4a) the evolution of desired vehicle density being in a mixed regime is illustrated. The results of achieving this state with and without feedback are shown on the plots below 4 (c) - (f). Thereby, the left column shows the evolution of state, whereas on the right column one can see the evolution of the absolute difference between the real and the desired states. Plots (c) and (d) illustrate the result if no feedback is applied on the boundaries, while plots (e) and (f) depict the situation if feedback with gains $k=0.1$ is applied. The corresponding error behaviour for different gains is shown on plot (b).

Comparing the plots we can see that the control including the feedback term performs considerably better. Without feedback the congested regime almost completely occupies the domain as time runs, while the feedback term makes the system approach the desired state after the time inferior to the minimal controllability time, which is $T_{c}=\frac{L}{v_{f}}+\frac{L}{|w|}=200.5 \mathrm{~s}$.

Note that an open-loop control (such as applying absorbing boundary conditions until the road becomes empty, and then applying desired inflows and outflows) will not achieve the goal at all due to the difference in initial densities (18).

\section{CONCLUSIONS}

We have designed boundary control laws in order to track a target space- and time-dependent trajectory. Physically, the real and the desired states correspond to the vehicle density. Both states are the solutions of LWR PDE with triangular fundamental diagram and are allowed to be in the mixed regime. This means that our controller allows us to drive any state, being in a partly congested partly free-flow regime or even being completely congested, to some desired state being in any regime we want. Achieving such a goal is possible using a feedback term, which physically corresponds to the difference between the number of vehicles in our system and in the desired one multiplied by some controller gain. This result was obtained by using the explicit solution formula to $\mathrm{H}-\mathrm{J}$, which enabled us to formulate and analyse the control restriction functions. The explicit solution was obtained using the properties of triangular FD due to the convenient shape of its Legendre transform. The numerical example verified the results and illustrated that feedback is absolutely necessary to achieve the goal.

For the future studies it might be challenging to extend this result to a large-scale, i.e., controlling the vehicle density $\rho_{d_{0}}(t)=0.04+0.04 \sin (t / 8)$ and $\rho_{d_{L}}(t)=0.1+0.06 \sin (t / 4)$, being in any regime in the whole city. Moreover, it could be 

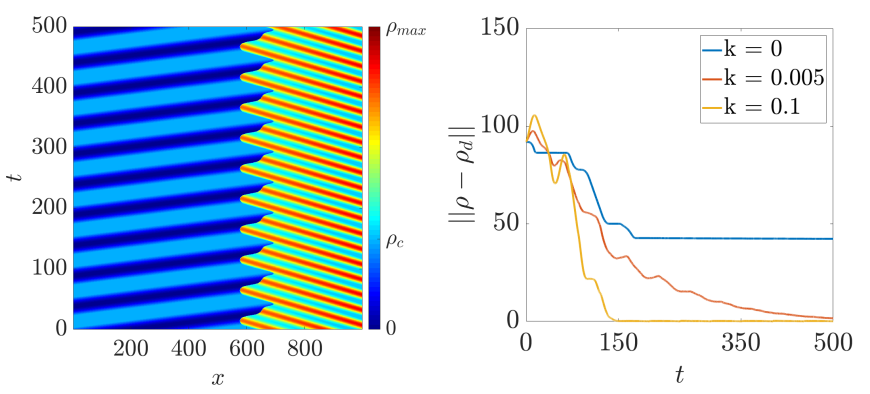

(a) $\rho_{d}(x, t)$

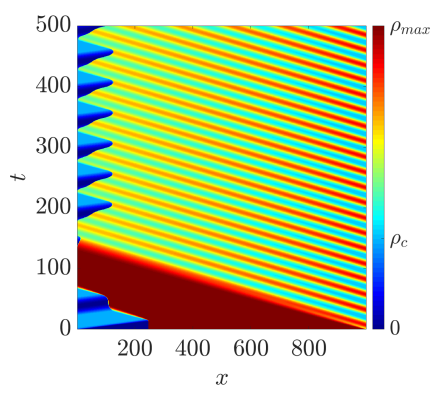

(c) $\rho(x, t)$, no feedback

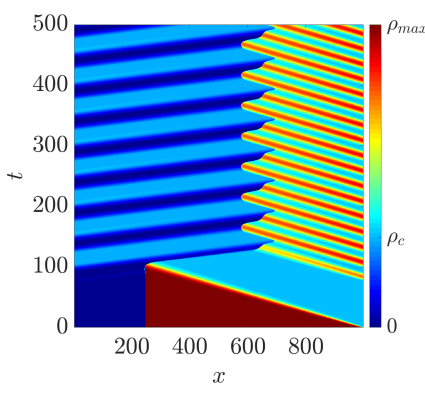

(e) $\rho(x, t), k=0.1$

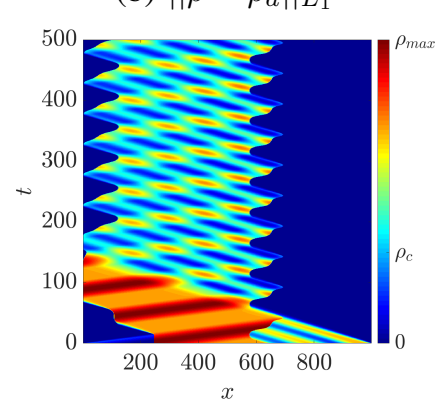

(d) $\left|\rho-\rho_{d}\right|$, no feedback

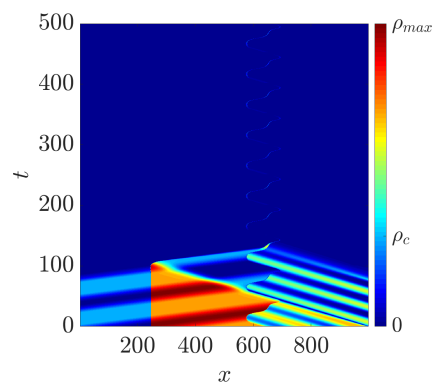

(f) $\left|\rho-\rho_{d}\right|, k=0.1$

Fig. 4. (a) Desired profile in space-time, (b) $\boldsymbol{L}_{1}$ error as a function of time for different control gains. Spatio-temporal evolution of the density (left) and of the absolute difference between the real and the target state (right) for: (c),(d) $k=0$; (e),(f) $k=0.1$.

interesting to derive an explicit solution for a H-J PDE for other shapes of fundamental diagram.

\section{APPENDIX I \\ SOLUTION OF HAMILTON-JACOBI EQUATION}

For a triangular FD the convex transform of FD (12) yields

$$
L(v)=\phi_{\max }-\rho_{c} v \quad \forall v \in\left[-w, v_{f}\right] .
$$

Being an integral function, $M(x, t)$ is defined up to a constant. Let us choose it such that $M(L, 0)=0$. From the geometrical interpretation and by integrating (8), we can equivalently write $\forall(x, t) \in[0, L] \times \mathbb{R}^{+}$

$$
M(x, t)=\int_{0}^{t} \phi_{\text {out }}(\tau) d \tau+\int_{x}^{L} \rho(s, t) d s,
$$

or

$$
M(x, t)=\int_{0}^{L} \rho_{0}(s) d s+\int_{0}^{t} \phi_{i n}(\tau) d \tau-\int_{0}^{x} \rho(s, t) d s .
$$

Now we can determine the value condition function which implies the calculation of $M_{\mathrm{Up}}(t), M_{\text {Down }}(t)$ and $M_{\text {Ini }}(x)$ :

$$
\begin{gathered}
M_{\mathrm{Up}}(t)=c(0, t)=\int_{0}^{t} \phi_{\text {in }}(\tau) d \tau+\int_{0}^{L} \rho_{0}(s) d s . \\
M_{\text {Down }}(t)=c(L, t)=\int_{0}^{t} \phi_{\text {out }}(\tau) d \tau . \\
M_{\text {Ini }}(x)=c(x, 0)=\int_{x}^{L} \rho_{0}(s) d s .
\end{gathered}
$$

Using these functions and the Lax-Hopf formula (13), we can restate the solution to (10) as a minimum of three new functions $M_{\mathrm{Up}}(x, t), M_{\text {Down }}(x, t)$ and $M_{\text {Ini }}(x, t)$ depending on both space and time, which are solutions to the Lax-Hopf formula associated only to one boundary or initial condition. For example, $M_{\mathrm{Up}}(x, t)$ is obtained by

$$
\begin{aligned}
M_{\mathrm{Up}}(x, t)= & \inf \{c(x-T v, t-T)+T L(v)\} \\
& \text { s.t. } \quad(v, T) \in\left[-w, v_{f}\right] \times \mathbb{R}^{+} \\
& \text {and } \quad(x-T v, t-T) \in\{0\} \times \mathbb{R}^{+},
\end{aligned}
$$

and the same formula yields $M_{\text {Down }}(x, t)$ for $(x-T v, t-T) \in$ $\{L\} \times \mathbb{R}^{+}$and $M_{\text {Ini }}(x, t)$ for $(x-T v, t-T) \in[0, L] \times\{0\}$.

By properties of infimum the original Lax-Hopf formula can thus be rewritten as

$$
M(x, t)=\min \left(M_{\mathrm{Ini}}(x, t), M_{\mathrm{Up}}(x, t), M_{\text {Down }}(x, t)\right) .
$$

Now we calculate all these functions explicitly. Note that we consider the solutions only for large time. Thus, in the following we will write the solutions only for

$$
t \geq \max \left(\frac{x}{v_{f}}, \frac{L-x}{w}\right) \text {. }
$$

\section{A. Upstream Boundary Condition}

The solution $M_{\mathrm{Up}}(x, t)$ is related to the number of vehicles that originate from the boundary $x_{s}=0$ and start travelling at $t_{s}:=t-T$. By looking at (11), we establish that $c(x-$ $T v, t-T)=M_{\mathrm{Up}}(t-T)$ in (56). The equality to zero of the first argument of c is provided by $T=\frac{x}{v}$, where $v \in\left[-w, v_{f}\right]$. Since $T$ is positive by definition, the minimal value of the time interval should be $T_{\min }=\frac{x}{v_{f}}$, which is consistent with (58). Using (50), this yields the following problem:

$$
M_{\mathrm{Up}}(x, t)=\inf _{T \in\left[\frac{x}{v_{f}},+\infty\right]}\left[M_{\mathrm{Up}}(t-T)+T \phi_{\max }\right]-x \rho_{c} .
$$

Using (53), the infimum problem reads

$$
\begin{aligned}
M_{\mathrm{Up}}(x, t)= & \inf _{T \in\left[\frac{x}{v_{f}},+\infty\right]}\left[\int_{0}^{t-T} \phi_{i n}(\tau) d \tau+T \phi_{\max }\right] \\
& +\int_{0}^{L} \rho_{0}(s) d s-x \rho_{c}
\end{aligned}
$$


which by using $T \phi_{\max }=\int_{t-T}^{T} \phi_{\max } d \tau$ can be rewritten as

$$
\begin{aligned}
M_{\mathrm{Up}}(x, t)= & \inf _{T \in\left[\frac{x}{v_{f}},+\infty\right]}\left[\int_{t-T}^{t}\left(\phi_{\max }-\phi_{i n}(\tau)\right) d \tau\right] \\
& +\int_{0}^{L} \rho_{0}(s) d s+\int_{0}^{t} \phi_{i n}(\tau) d \tau-x \rho_{c},
\end{aligned}
$$

The infimum is provided by $T^{*}=T_{\min }=\frac{x}{v_{f}}$, i.e. the correct solution is related to the path along which the vehicle has the maximal velocity. Substituting $T=T_{\min }$, we get

$$
M_{\mathrm{Up}}(x, t)=\int_{0}^{t-\frac{x}{v_{f}}} \phi_{i n}(\tau) d \tau+\int_{0}^{L} \rho_{0}(s) d s .
$$

\section{B. Downstream Boundary Condition}

Further, we need to calculate $M_{\text {Down }}(x, t)$, which is related to the downstream boundary $x_{s}=L$. The calculation is done performing the same steps as for $M_{\mathrm{Up}}(x, t)$, and we obtain

$$
M_{\text {Down }}(x, t)=\int_{0}^{t-\frac{L-x}{w}} \phi_{\text {out }}(\tau) d \tau+\rho_{\max }(L-x) .
$$

\section{Initial Condition}

Finally, we calculate the function $M_{\text {Ini }}(x, t)$ determined by the vehicle with known label at $t_{s}=0$ (55). The equality to zero of the time argument in the value condition function is provided by $t=T$. This yields

$$
M_{\text {Ini }}(x, t)=\inf _{v \in\left[-w, v_{f}\right]}\left[M_{\text {Ini }}(x-t v)-t \rho_{c} v\right]+t \phi_{\max }
$$

Using the definition of $M_{\text {Ini }}(x)$ from (55), we obtain

$$
M_{\text {Ini }}(x, t)=\inf _{v \in\left[-w, v_{f}\right]}\left[\int_{x-t v}^{L} \rho_{0}(s) d s-t \rho_{c} v\right]+t \phi_{\max } .
$$

We decompose the integral as $\int_{x-t v}^{L} \rho_{0}(s) d s=\int_{x}^{L} \rho_{0}(s) d s+$ $\int_{x-t v}^{x} \rho_{0}(s) d s$, and the second term as $-t \rho_{c} v=\int_{x-t v}^{x}-\rho_{c} d s$ :

$M_{\text {Ini }}(x, t)=\int_{x}^{L} \rho_{0}(s) d s+t \phi_{\max }+\inf _{v \in\left[-w, v_{f}\right]} \int_{x-t v}^{x}\left(\rho_{0}(s)-\rho_{c}\right) d s$.

We define $y=x-t v$ and take the infimum over $y$ :

$$
M_{\text {Ini }}(x, t)=\int_{x}^{L} \rho_{0}(s) d s+t \phi_{\max }+\inf _{y \in\left[x-t v_{f}, x+t w\right]} \int_{y}^{x}\left(\rho_{0}(s)-\rho_{c}\right) d s .
$$

Note that the coordinates can not lie outside the interval $[0, L]$ :

$$
M_{\text {Ini }}(x, t)=\int_{x}^{L} \rho_{0}(s) d s+t \phi_{\max }+\inf _{y \in[0, L]} \int_{y}^{x}\left(\rho_{0}(s)-\rho_{c}\right) d s .
$$

\section{Unique Solution $\mathrm{M}(\mathrm{x}, \mathrm{t})$}

To obtain a unique solution, we insert the results from (59), (60) and (61) in (57), and we get the minimum function. However, we still need to solve one infinum problem due to (61), which requires information on crossings of the critical value by the density at initial condition. In (61) the only time depending term is $t \phi_{\max }$, thus $d M_{\text {Ini }} / d t=\phi_{\max }$. Taking the time derivative for other terms yields $d M_{\mathrm{Up}} / d t=\phi_{\text {in }}(t-$ $\left.x / v_{f}\right)$ and $d M_{\text {Down }} / d t=\phi_{\text {out }}(t-(L-x) / w)$. By $\phi(\rho(\cdot, \cdot)) \leq$ $\phi_{\max }$ we see that except for some special cases $M_{\text {Ini }}$ grows faster than the others. In Appendix II we have estimated the minimal time $t_{\min }(67)$ needed for initial conditions to leave the system Thus, $M_{\text {Ini }}$ can be excluded from the minimum operator $\forall t \in\left[t_{\text {min }},+\infty\right)$ :

$$
\begin{array}{r}
M(x, t)=\min \left\{\int_{0}^{t-\frac{x}{v_{f}}} \phi_{\text {in }}(\tau) d \tau+\int_{0}^{L} \rho_{0}(s) d s,\right. \\
\left.\int_{0}^{t-\frac{L-x}{w}} \phi_{\text {out }}(\tau) d \tau+\rho_{\max }(L-x)\right\} .
\end{array}
$$

\section{APPENDIX II}

\section{TIME FOR NEGLECTING THE INITIAL CONDITIONS}

We want to find the minimal time $t_{\text {min }}$, after which the initial conditions have definitely left the system, i.e., $\forall(x, t) \in[0, L] \times\left[t_{\text {min }},+\infty\right): M_{\mathrm{Ini}}(x, t) \geq M_{\mathrm{Up}}(x, t)$ or $M_{\text {Ini }}(x, t) \geq M_{\text {Down }}(x, t)$. Let us first establish the time for which $M_{\text {Ini }}(x, t) \geq M_{\mathrm{Up}}(x, t)$, then we will do the same for $M_{\text {Down }}(x, t)$, and then the final value will be the minimum from of cases.

Thus, using $M_{\mathrm{Up}}(x, t)$ from (59) and $M_{\mathrm{Ini}}(x, t)$ from (61) $t-\frac{x}{v_{f}}$

with $t \phi_{\max }=\int_{0} \phi_{\max } d \tau+\frac{x}{v_{f}} \phi_{\max }$, we can write $\forall(x, t) \in$ $[0, L] \times\left[\max \left(\frac{x}{v_{f}}, \frac{L-x}{w}\right),+\infty\right)$ that

$$
\begin{aligned}
& M_{\mathrm{Ini}}(x, t)-M_{\mathrm{Up}}(x, t)=\int_{0}^{t-\frac{x}{v_{f}}}\left(\phi_{\max }-\phi_{\text {in }}(\tau)\right) d \tau+ \\
& \frac{x}{v_{f}} \phi_{\max }-\int_{0}^{x} \rho_{0}(s) d s+\inf _{y \in[0, L]} \int_{y}^{x}\left(\rho_{0}(s)-\rho_{c}\right) d s .
\end{aligned}
$$

We can estimate an upper bound of (62) by using:

$$
\begin{aligned}
& \frac{x}{v_{f}} \phi_{\text {max }} \geq 0, \quad-\int_{0}^{x} \rho_{0}(s) d s \geq-\int_{0}^{L} \rho_{\max } d s, \\
& \text { and } \inf _{y \in[0, L]} \int_{y}^{x}\left(\rho_{0}(s)-\rho_{c}\right) d s \geq-\int_{0}^{L} \rho_{c} d s,
\end{aligned}
$$


and, thus, we get

$$
\begin{aligned}
M_{\mathrm{Ini}}(x, t) & -M_{\mathrm{Up}}(x, t) \geq \int_{0}^{t-\frac{x}{v_{f}}}\left(\phi_{\max }-\phi_{\text {in }}(\tau)\right) d \tau \\
& -\int_{0}^{L}\left(\rho_{\max }+\rho_{c}\right) d s
\end{aligned}
$$

Further, we use Assumption 2 to make the following bound:

$$
\int_{0}^{t-\frac{x}{v_{f}}}\left(\phi_{\max }-\phi_{i n}(\tau)\right) d \tau \geq\left\lfloor\frac{t-\frac{x}{v_{f}}}{T}\right\rfloor \varepsilon
$$

where $\varepsilon>0$ and $T=\min \left\{\frac{L}{v_{f}}, \frac{L}{w}\right\}$. Thus, we can further bound (63) by:

$$
M_{\text {Ini }}(x, t)-M_{\mathrm{Up}}(x, t) \geq\left\lfloor\frac{t-\frac{L}{v_{f}}}{T}\right\rfloor \varepsilon-\int_{0}^{L}\left(\rho_{\max }+\rho_{c}\right) d s
$$

Finally, we can determine for which $t$ the expression (64) is non-negative:

$$
t \geq \frac{L}{v_{f}}+\left\lceil\frac{1}{\varepsilon} \int_{0}^{L}\left(\rho_{\max }+\rho_{c}\right)\right\rceil \min \left\{\frac{L}{v_{f}}, \frac{L}{w}\right\}
$$

Following the same steps, we obtain a similar result for $t$ such that $M_{\text {Ini }}(x, t)-M_{\text {Down }}(x, t) \geq 0$ :

$$
t \geq \frac{L}{w}+\left\lceil\frac{1}{\varepsilon} \int_{0}^{L}\left(\rho_{\max }+\rho_{c}\right)\right\rceil \min \left\{\frac{L}{v_{f}}, \frac{L}{w}\right\}
$$

The combination of (65) and (66) yields the lowest time bound for the initial conditions to leave the system:

$$
t_{\min }=\min \left\{\frac{L}{v_{f}}, \frac{L}{w}\right\}\left(1+\left\lceil\frac{1}{\varepsilon} \int_{0}^{L}\left(\rho_{\max }+\rho_{c}\right)\right\rceil\right) .
$$

\section{APPENDIX III}

\section{NECESSARY CONDITIONS FOR TRACKING DESIRED STATE}

In accordance with Problem 1, we should find $u_{i n}(t)$ and $u_{\text {out }}(t)$ such that the asymptotic equality of $M(x, t)$ and $M_{d}(x, t)$ up to some constant $M_{0}$ is guaranteed. For the equality of two min functions (14) and (15), it is suffices to provide the equality of their arguments, thus $\forall(x, t) \in$
$[0, L] \times\left[t_{\min },+\infty\right)$ we get

$$
\begin{aligned}
\int_{0}^{t-\frac{x}{v_{f}}} \phi_{i n_{d}}(\tau) d \tau+\int_{0}^{L} \rho_{d_{0}}(s) d s+M_{0} \\
=\int_{0}^{t-\frac{x}{v_{f}}} \phi_{i n}(\tau) d \tau+\int_{0}^{L} \rho_{0}(s) d s,
\end{aligned}
$$

$$
\begin{aligned}
& \int_{0}^{t-\frac{L-x}{w}} \phi_{\text {out }_{d}}(\tau) d \tau+\rho_{\max }(L-x)+M_{0} \\
& =\int_{0}^{t-\frac{L-x}{w}} \phi_{\text {out }}(\tau) d \tau+\rho_{\max }(L-x) .
\end{aligned}
$$

Firstly, by taking the time derivative of (68), we see that in the steady-state $\phi_{\text {in }}(t) \equiv \phi_{i n_{d}}(t)$ and $\phi_{\text {out }}(t) \equiv \phi_{\text {out }}(t)$.

Secondly, by expressing $M_{0}$ from both parts of (68), we obtain the necessary condition (16) to track $\rho_{d}$.

\section{REFERENCES}

[1] B. D. Greenshields, "A study of traffic capacity", Proc. Highway Res. Board, vol. 14, pp. 448-477, 1935.

[2] M. Lighthill and G. Whitham, "On kinematic waves, II: A theory of traffic flow on long crowded roads", Proc. Royal Soc. London, vol. 229, no. 1178 , pp. $317-345,1956$.

[3] P. Richards, "Shock waves on the highway", Operations Res., vol. 47, no. 1 , pp. $42-51,1956$.

[4] S. K. Godunov, "A difference method for numerical calculation of discontinuous solutions of the equations of hydrodynamics", Matematicheskii Sbornik, vol. 47, no. 3, pp. 271-306, 1959.

[5] K. Moskowitz "Discussion of freeway level of service as influenced by volume and capacity characteristics by dr drew and cj keese" in Proc. Highway Research Record, 1965, vol. 99, pp. 43-44.

[6] R. Courant, K. Friedrichs and H. Lewy, "On the Partial Difference Equations of Mathematical Physics", IBM Journal of Research and Development, vol. 11, no. 2, 1967.

[7] E. Angel and R. Bellman, "Dynamic Programming and Partial Differential Equations", Academic Press, New York, NY, 1972.

[8] K. T. Joseph and G. D. Veerappa Godwa, "Explicit formula for the solution of convex conservation laws with boundary condition", Duke Math. Journ., vol. 62, no. 2, pp. 401-416, 1991.

[9] K. T. Joseph and G. D. Veerappa Godwa, "Solution of convex conservation laws in a strip", in Proc. of the Indian Academy of SciencesMathematical Sciences, vol. 102, no. 1, Springer India, 1992.

[10] G Newell, "A simplified theory of kinematic waves in highway traffic, part i,ii and iii", Trans. Res. B: Meth., vol. 27, no. 4, pp. 281-287, 1993.

[11] C. F. Daganzo, "The cell transmission model: A dynamic representation of highway traffic consistent with the hydrodynamic theory", Transportation Research Part B: Methodological, vol. 28, no. 4, pp. 269-287, 1994.

[12] L. C. Evans, Partial Differential Equations. Providence, RI: Graduate Studies in Mathematics. American Mathematical Society, 1998.

[13] A. Aw and M. Rascle, "Resurrection of "second order models" of traffic flow?", SIAM J Appl Math, vol. 60, no. 3, pp. 916-938, 2000.

[14] C. F. Daganzo, "A behavioral theory of multi-lane traffic flow. Part I: long homogeneous freeway sections", Transportation Research Part B: Methodological, vol. 36, no. 2, pp. 131-158, 2002.

[15] S. Ulbrich, "A Sensitivity and Adjoint Calculus for Discontinuous Saolutions of Hyperbolic Conservation Laws with Source Terms", J. Control Optim., vol. 41, no. 3, pp. 740-797, 2002.

[16] G. C. Wong and S. C. Wong, "A multi-class traffic flow model: an extension of LWR model with heterogeneous drivers", Transportation Research Part A: Policy and Practice, vol. 36, no. 9, pp. 827-841, 2002.

[17] S. Benzoni-Gavage and R. M. Colombo, "An $n$-populations model for traffic flow", Eur. J. Appl. Math., vol. 14, no. 5, pp. 587-612, 2003.

[18] J. M. Greenberg, A. Klar and M. Rascle, "Congestion on multilane highways", SIAM J. Appl. Math., vol. 63, no. 3, pp. 818-833, 2003. 
[19] C. F. Daganzo, "A variational formulation of kinematic waves: basic theory and complex boundary conditions", Transportation Research Part B: Methodological, vol. 39, no. 2, pp. 187-196, 2005.

[20] M. Gugat, M. Herty, A. Klar and G. Leugering, "Optimal control for traffic flow networks", J. Opt. Th. Appl., vol. 126 (3), pp. 589-616, 2005.

[21] C. F. Daganzo, "On the variational theory of traffic flow: Well-posedness, duality and applications", Netw. Heter. Med., vol. 1, pp. 601-619, 2006.

[22] D. Jacquet, M. Krstic and C. Canudas-de-Wit, "Optimal control of scalar one-dimensional conservation laws", in Proc. 25 th $A C C$, Minneapolis, MN, USA, 2006, pp. 5213-5218.

[23] I. Strub and A. Bayen, "Weak formulation of boundary conditions for scalar conservation laws: An application to highway traffic modelling", Int. J. Robust Nonlinear Control, vol. 16, no. 4, pp. 733-748, 2006.

[24] J-P. Aubin, A. Bayen and P. Saint-Pierre, "Dirichlet problems for some Hamilton-Jacobi equations with inequality constraints", SIAM J. Control Optim., vol. 47, no. 5, pp. 2348-2380, 2008.

[25] M. Z. F. Li, "A generic characterization of equilibrium speed-flow curves", Transportation Science, vol. 42, no. 2, pp. 220-235, 2008.

[26] S. Blandin, X. Litrico and A. Bayen, "Boundary stabilization of the inviscid burgers equation using a Lyapunov method", in Proc. $49^{\text {th }}$ IEEE Conf. Decis. Control (CDC), 2010, pp. 1705-1712.

[27] T. Li and B. Rao, "Strong (Weak) Exact Controllability and Strong (Weak) Exact Observability for Quasilinear Hyperbolic Systems", Chin. Ann. Math., vol. 31 B, no. 5, pp. 723-742, 2010.

[28] C. G. Claudel and A. M. Bayen, "Lax-Hopf based incorporation of internal boundary conditions into Hamilton-Jacobi equation. Part I: Theory", IEEE TAC, vol. 55, no. 5, pp. 1142-1157, 2010.

[29] C. G. Claudel and A. M. Bayen, "Lax-Hopf based incorporation of internal boundary conditions into Hamilton-Jacobi equation. Part I: Computational methods", IEEE TAC, vol. 55, no. 5, pp. 1158-1174, 2010.

[30] R. Carlson, I. Papamichail and M.Papageorgiou, "Local feedback-based mainstream traffic flow control on motorways using variable speed limits", IEEE Trans. Intel. Trans. Sys., vol. 12, no. 4, pp. 1261-1276, 2011.

[31] R. M. Colombo, P. Goatin and M. D. Rossini, "On the modeling and management of traffic", ESAIM:M2AN, vol. 45, pp. 853-872, 2011.

[32] P. E. Mazaré, A. H. Dehwah, C. G. Claudel and A. M. Bayen, "Analytical and grid-free solutions to the Lighthill-Whitham-Richards traffic model", Trans. Res. B: Method., vol. 45, no. 10, pp. 1727-1748, 2011.

[33] J. M Coron, R. Vazquez, M. Krstic and G. Bastin, "Local exponential $\mathrm{H}_{2}$ stabilization of a $2 \times 2$ quasilinear hyperbolic system using backstepping", SIAM J. Control Optim., vol. 51, no. 3, 2011, pp. 2005-2035.

[34] Y. Li, E. Canepa and C. Claudel, "Optimal Control of Scalar Conservation Laws Using Linear/Quadratic Programming: Application to Transportation Networks", IEEE Trans. Contr. Net. Sys., vol. 1, no. 1, pp. 28-39, 2014

[35] Y. Li, E. Canepa and C. Claudel, "Optimal traffic control in highway transportation networks using linear programming", in 2014 European Control Conference (ECC), June 24-27, 2014.

[36] C. C. de Wit, F. Morbidi, L. L. Ojeda, A. Y. Kibangou, I. Bellicot and P. Bellemain, "Grenoble Traffic Lab: An Experimental Platform for Advanced Traffic Monitoring and Forecasting [Applications of Control]", IEEE Cont. Syst. Magazine, vol. 35, no. 3, pp. 23-39, 2015.

[37] J. D. Reilly, S. Samaranayake, M. L. Delle Monache, W. Krichene, P. Goatin, and A. M. Bayen, "Adjoint-based optimization on a network of discretized scalar conservation laws with applications to coordinated ramp metering", Journal of Optimization Theory and Applications, vol. 167, no. 2, pp. 733-760, 2015.

[38] Y. Zu, R. Dai and J. Dong, "Convex Optimization for Energy-Efficient Traffic Control", in IEEE 55th Conf. Dec. and Contr. (CDC), 2016.

[39] S. Blandin, X. Litrico, M. L. Delle Monache, B. Piccoli and A. Bayen, "Regularity and Lyapunov Stabilization of Weak Entropy Solutions to Scalar Conservation Laws", IEEE TAC, vol. 62, no. 4, 2017.

[40] Y. Zhang and P. A. Ioannou, "Coordinated variable speed limit, ramp metering and lane change control of highway traffic", IFAC-PapersOnLine, vol. 50, no. 1, pp. 5307-5312, 2017.

[41] M. Simoni and C. G. Claudel, "A Fast Lax-Hopf formula to solve the Lighthill-Whitham-Richards traffic flow model on networks", arXiv preprint arXiv:1802.05391, 2018.

[42] G. Bastin, J. M. Coron, A. Hayar and P. Shang, "Exponential boundary feedback stabilization of a shock steady state for the inviscid Burgers equation", Math. Mod. Meth. Appl. Sci., vol. 29 (2), pp. 271-316, 2019.

[43] N. Bekiaris-Liberis and R. Vazquez, "Nonlinear bilateral outputfeedback control for a class of viscous Hamilton-Jacobi PDEs", $A u$ tomatica, vol. 101, pp. 223-231, 2019.
[44] C. Donadello and V. Perrolaz, "Exact controllability to trajectories for entropy solutions to scalar conservation laws in several space dimensions", C. R. Acad. Sci. Paris, vol. 357, no. 3, pp. 263-271, 2019.

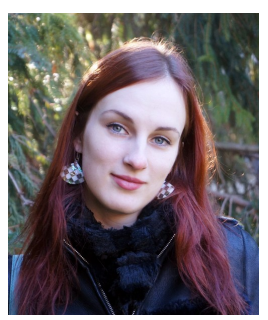

Liudmila Tumash received both the B.Sc. and the M.Sc. degree in Institute of Theoretical Physics from the Technical University of Berlin, Berlin, Germany, specialising on control of chaotic networks. She was the recipient of Physik-Studienpreis for the excellent Master's degree.

She is currently a doctoral researcher at CNRS, Gipsa-Lab, Grenoble, France. Her research mainly focuses on control of traffic systems in large-scale networks.

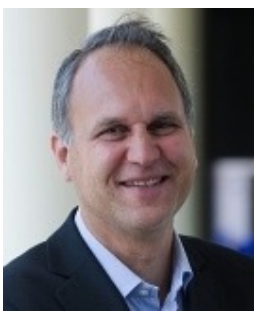

Carlos Canudas-de-Wit (F'16) was born in Villahermosa, Mexico, in 1958. He received the B.S. degree in electronics and communications from the Monterrey Institute of Technology and Higher Education, Monterrey, Mexico, in 1980, and the M.S. and Ph.D. degrees in automatic control from the Department of Automatic Control, Grenoble Institute of Technology, Grenoble, France, in 1984 and 1987, respectively.

$\mathrm{He}$ is currently a Directeur de recherche (Senior Researcher) with CNRS, Grenoble, where he is the Leader of the NeCS Team, a joint team of GIPSA-Lab (CNRS) and INRIA, on networked controlled systems.

Dr. Canudas-de-Wit is an IFAC Fellow. He was an Associate Editor of the IEEE Transactions on Automatic Control, the Automatica, the IEEE Transactions on Control Systems Technology. He is an Associate Editor of the Asian Journal of Control, and the IEEE Transactions on Control of Network Systems. He served as the President of the European Control Association from 2013 to 2015, and a member of the IEEE Board of Governors of the Control System Society from 2011 to 2014. He holds the ERC Advanced Grant Scale-FreeBack from 2016 to 2021.

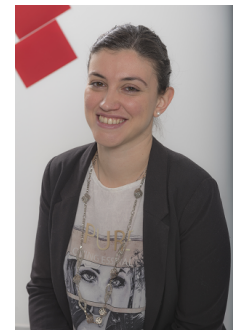

Maria Laura Delle Monache is a research scientist in the Networked Controlled Systems team at Inria and in GIPSA-Lab (Department of Control) in Grenoble. She received the B.S. degree in industrial engineering from the University of L'Aquila, L'Aquila, Italy, the M.S. degree in mathematical engineering from the University of L'Aquila, and the University of Hamburg, Germany, and the Ph.D. degree in applied mathematics from the University of Nice-Sophia Antipolis, Nice, France in 2009, 2011, and 2014, respectively. Prior to Inria, she was a Postdoctoral researcher at Rutgers University Camden. Her research interest is mainly related to the mathematical and engineering aspects of traffic flow. In particular, she is interested in mathematical modeling and control of traffic flow applications. 